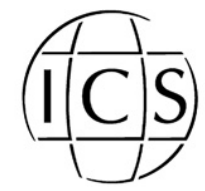

www.ics-elsevier.com

\title{
Numerical approaches for dipole modeling in finite element method based source analysis
}

\author{
C.H. Wolters ${ }^{\mathrm{a}, \mathrm{b}, *}$, H. Köstler ${ }^{\mathrm{b}, \mathrm{c}}$, C. Möller ${ }^{\mathrm{c}}$, \\ J. Härdtlein ${ }^{\mathrm{c}}$, A. Anwander ${ }^{\mathrm{d}}$ \\ ${ }^{a}$ Institute for Biomagnetism and Biosignalanalysis, Münster University Hospital, Münster, Germany \\ b Scientific Computing and Imaging Institute, University of Utah, Salt Lake City, USA \\ ${ }^{c}$ Department of Computer Science 10, University Erlangen-Nuremberg, Erlangen, Germany \\ d Max Planck Institute for Human Cognitive and Brain Sciences, Leipzig, Germany
}

\begin{abstract}
In EEG/MEG source analysis, a mathematical dipole is widely used as the "atomic" structure of the primary current distribution. When using realistic finite element models for the forward problem, the current dipole introduces a singularity on the right-hand side of the governing differential equation that has to be treated specifically. We evaluated and compared three different numerical approaches, a subtraction method, a direct approach using partial integration and a direct approach using the principle of Saint Venant. Evaluation and comparison were carried out in a fourlayer sphere model using quasi-analytical formulas. (C) 2007 Elsevier B.V. All rights reserved.
\end{abstract}

Keywords: EEG; MEG; Source analysis; Dipole; Finite Element method; Singularity treatment; Subtraction method; Direct potential approach

\section{Introduction}

An important aspect in Finite Element (FE) method based volume conductor modelling in EEG/MEG source analysis is the way of modelling the current dipole. We developed and implemented three different numerical approaches,

a) a subtraction potential method $[1,5,7,11]$

b) a direct potential approach using Partial Integration [6,8] and

c) a direct potential approach using Saint Venant's principle $[2,9,10]$.

\footnotetext{
* Corresponding author. Malmedyweg 15, 48149 Münster, Germany. Tel.: +49 25183 56904; fax: +49 25183 56874.

E-mail address: carsten.wolters@uni-muenster.de (C.H. Wolters).

0531-5131/ (C) 2007 Elsevier B.V. All rights reserved. doi:10.1016/j.ics.2007.02.014
} 
In this paper, we evaluate and compare the different techniques with regard to their accuracy in a four-layer sphere model [3].

\section{Theory}

a) The subtraction potential approach divides the total potential into a singularity potential (dipole in infinite region of homogeneous conductivity) and a correction potential. When subtracting the differential equation for the singularity potential from the starting potential equation, a singularity free Poisson-problem with inhomogeneous Neumann boundary conditions for the correction potential results. Our 3D FE approach for anisotropic head models is closely related to the 2D implementation in [1]. We additionally performed a numerical analysis with a correction potential existence and uniqueness proof and FE convergence properties [11].

b) Partial Integration can be used on the right-hand-side (RHS) of the starting Poissonlike potential equation in the variational FE formulation. The RHS is then identical to an evaluation of the scalar product of the dipole moment with the gradient of the basis-function evaluated at the source position. For linear Ansatz-functions, the gradient is constant and non-zero only over the source element, so that the resulting FE linear equation system has only 8 non-zero RHS entries (identical to monopolar loads) when using hexahedra elements. This approach was used, e.g., in $[6,8]$.

c) Saint Venant's principle states that the specific (fine) details of load application do not influence the results observed in some distance away from the locus of load application. Following [2], a dipole can be modeled by placing monopolar sources on all neighboring FE nodes to that FE node which is closest to the source. By means of solving a local Tikhonov-Phillips regularization problem, the monopolar loads are computed so that, multiplied with their "lever arms" (distance of the node to the source), the dipole moment is optimally matched.

\section{Method}

The quasi-analytical series expansion formulas [3] were used as a basis for our accuracy studies. A four-layer sphere model (radii 92, 86, 80, $78 \mathrm{~mm}$ with conductivities of 0.33 , $0.0042,1.0,0.33 \mathrm{~S} / \mathrm{m}$, respectively) was discretized with a $2 \mathrm{~mm}$ regular hexahedra mesh (426 K nodes, $406 \mathrm{~K}$ elements) using the software VGRID (http://www.simbio.de). For all forward EEG simulations, the software NeuroFEM-COLSAMM (http://www.simbio.de, see [4] for COLSAMM) was used with linear FE Ansatz-functions and an Algebraic MultiGrid preconditioned Conjugate Gradient (AMG-CG) method for solving the resulting FE linear equation systems up to a relative accuracy of $10^{-8}$ [9]. 134 electrodes were distributed in a most regular way over the outer sphere surface. The topography error Relative Difference Measure (RDM) and the MAGnification error (MAG) $[5,7,11]$ between quasi-analytic and numeric results at those measurement sensors were evaluated for dipoles with fixed $x$ and $z$ and varying (in $1 \mathrm{~mm}$ steps) $y$-coordinate (depths) and either tangential or radial orientation. The eccentricity was limited to a percentage of the inner layer depending on the number of compartments, because it can be expected that the dipole is at least $2 \mathrm{~mm}$ below the surface in the middle of the gray matter compartment. Dipole strengths of $1 \mathrm{nAm}$ were used. 


\section{Results}

As Fig. 1 shows, one important advantage of the subtraction approach over the direct methods was that the error curves (and thus the cost functions during inverse optimization) were smooth, while the accuracy of both Venant's and Part.Int. direct potential methods were oscillating. While Venant performed best for sources on FE nodes (i.e. $x, y$ and $z$ are even numbers in Fig. 1), Part.Int. performed best if the source is positioned in the center of an element ( $x, y$ and $z$ odd numbers). On the other hand, the subtraction approach with linear basis-functions was computationally more expensive and more sensitive to conductivity jumps in source vicinity if the source was pointing towards the jump (Fig. 1, radial sources). For the subtraction approach, highest relative AMG-CG solver accuracies were needed for the most eccentric sources with $10^{-4}$ being sufficient for the whole eccentricity range [11].
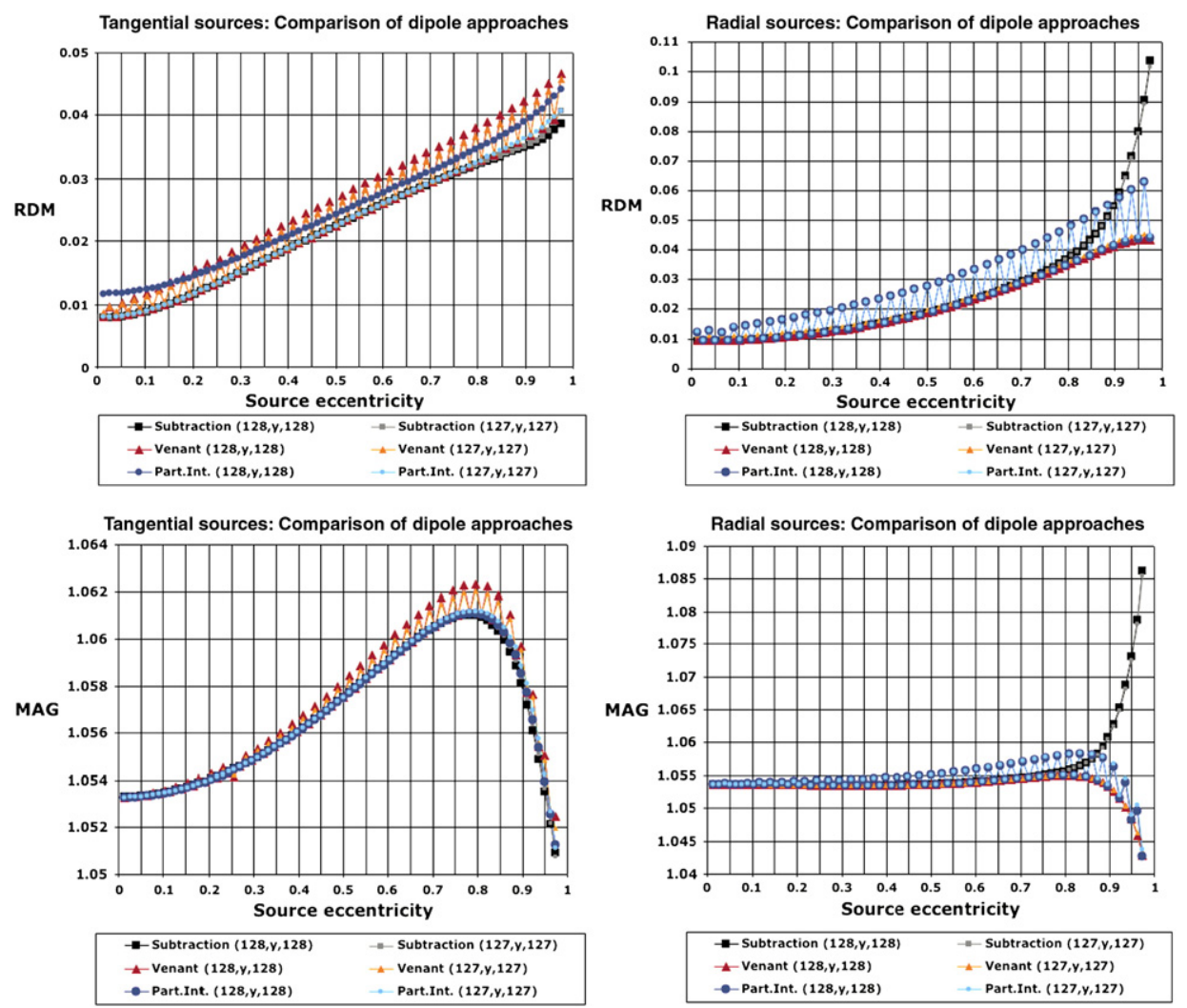

Fig. 1. Accuracy in $2 \mathrm{~mm}$ regular hexahedra FE model of the four compartment sphere: RDM (top row) and MAG (bottom row) for tangentially (left) and radially (right) oriented sources for the three FE forward modeling techniques subtraction (cubes: black, gray), Venant (triangles: red, orange) and Partial Integration (spheres: dark and light blue). Dipoles with fixed $x$ and $z$ and varying $y$-coordinate at realistic source eccentricities of 0 to $97 \%$ of the inner compartment at either $(128, y, 128)$ (along nodes and faces) or $(127, y, 127)$ (through element barycenters) were examined. (For interpretation of the references to colour in this figure legend, the reader is referred to the web version of this article.) 


\section{Conclusions}

Inverse source analysis: All presented numeric approaches could exploit the computationally efficient EEG/MEG lead field bases concept which reduced the "number of FE equation systems to solve" to the "number of sensors" [8,9]. Each FE based forward solution was then especially cheap for the direct potential methods [9]. With our current implementation, we recommend the choice of the Venant direct potential approach at least for those inverse methods exploiting influence matrices (beamformer, current density approaches, scanning methods). The direct potential approaches are less appropriate for inverse optimization methods in continuous parameter space (e.g., dipole fits using simplex optimization), because of the presented error curve oscillations, there might be a higher risk to get stuck in local minima.

Anisotropy: All three FE approaches can treat remote tissue anisotropy (skull, white matter) [10], but a clear theory for local anisotropy (gray matter) only exists for the subtraction approach [11].

Perspective: The subtraction method is theoretically best understood and bears the highest future potential. An improved numerical quadrature should solve the accuracy problems when the source approaches a conductivity jump.

\section{Acknowledgement}

This research was supported by the German Research Foundation (DFG, WO 1425/1-1).

\section{References}

[1] K.A. Awada, et al., Computational aspects of finite element modeling in EEG source localization, IEEE Trans. Biomed. Eng. 44 (8) (1997) 736-751.

[2] H. Buchner, et al., Inverse localization of electric dipole current sources in finite element models of the human head, Electroencephalogr. Clin. Neurophysiol. 102 (4) (1997) 267-278.

[3] J.C. de Munck, M.J. Peters, A fast method to compute the potential in the multisphere model, IEEE Trans. Biomed. Eng. 40 (11) (1993) 1166-1174.

[4] J. Härdtlein, C. Pflaum, Efficient and User-friendly Computation of Local Stiffness Matrices, Frontiers in Simulation, Simulations technique, 18th Symposium in Erlangen, ISBN: 3-936150-41-9, Sep. 2005, pp. 748-753.

[5] G. Marin, et al., Influence of skull anisotropy for the forward and inverse problem in EEG: simulation studies using the FEM on realistic head models, Hum. Brain Mapp. 6 (1998) 250-269.

[6] Mohr, M. Simulation of Bioelectric Fields: The Forward and Inverse Problem of Electro-encephalographic Source Analysis. PhD thesis, Universität Erlangen-Nürnberg, Arbeitsberichte des Instituts für Informatik, 37 (6), ISSN 1611-4205, 2004.

[7] P.H. Schimpf, C.R. Ramon, J. Haueisen, Dipole models for the EEG and MEG, IEEE Trans. Biomed. Eng. 49 (5) (2002) 409-418.

[8] D. Weinstein, L. Zhukov, C. Johnson, Lead-field bases for electroencephalography source imaging, Ann. Biomed. Eng. 28 (9) (2000) 1059-1066.

[9] C.H. Wolters, L. Grasedyck, W. Hackbusch, Efficient computation of lead field bases and influence matrix for the FEM-based EEG and MEG inverse problem, Inverse Problems 20 (2004) 1099-1116.

[10] C.H. Wolters, et al., Influence of tissue conductivity anisotropy on EEG/MEG field and return current computation in a realistic head model: a simulation and visualization study using high-resolution finite element modeling, NeuroImage 30 (3) (2006) 813-826.

[11] Wolters, C.H.,Köstler, H. , Möller, C., Härdtlein, J., Grasedyck, L., and Hackbusch, W., Numerical mathematics for the modeling of a current dipole in EEG source analysis using finite element head models, SIAM J. Sci. Comp., in review, pp. 1-19, 2006. 